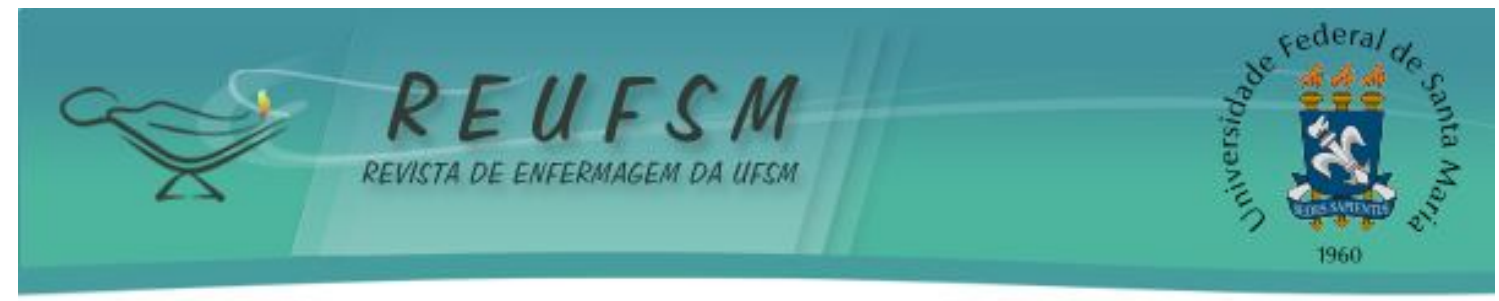

RELATO DE EXPERIÊNCIA

\title{
AMAMENTAÇÃO NA PRIMEIRA HORA DE VIDA EM UM HOSPITAL PRIVADO DE PORTO ALEGRE/RS - RELATO DE EXPERIÊNCIA
}

\author{
BREASTFEEDING AT THE FIRST HOUR OF LIFE IN A PRIVATE HOSPIT AL IN PORTO \\ ALEGRE, RS - EXPERIENCE'S REPORT
}

\section{AMAMENT ACIÓN EN LA PRIMERA HORA DE VIDA EN UN HOSPIT AL PRIVADO DE PORTO ALEGRE/RS - RELATO DE EXPERIENCIA}

\author{
Márcia Rejane Strapasson ${ }^{1}$ \\ Ana Carla dos Santos Fischer ${ }^{2}$ \\ Ana Lucia de Lourenzi Bonilha ${ }^{3}$
}

RESUMO: Objetivo: relatar a experiência da amamentação na primeira hora de vida do recém-nascido em um hospital privado de Porto Alegre/ RS abordando suas vantagens e as principais dificuldades para a adoção desta prática. Método: Trata-se de um relato de experiência realizado pelas enfermeiras durante 0 atendimento ao parto no centro obstétrico. Resultados: A experiência relatada permitiu dar visibilidade a temática da amamentação precoce, apontando para a importância desta prática na sala de parto. Verificou-se que esta medida não está implementada efetivamente, sendo postergada à sala de recuperação obstétrica. Há predominância de cesarianas na instituição hospitalar, o que dificulta a promoção do aleitamento materno no cenário do parto e nascimento. Outro fator que limita esta prática é a assistência presa às normas, às regras institucionais e à cultura da competência biomédica. Conclusão: Destaca-se a necessidade da instituição hospitalar desenvolver políticas de promoção e proteção ao al eitamento materno.

Descritores: Aleitamento materno; Enfermagem obstétrica; Parto humanizado; Saúde da mulher.

ABSTRACT: Objective: report the experience of breastfeeding at the newborn's first time of life in a private hospital in Porto Alegre, RS, addressing its advantages and the difficulties into the adoption of this approach. Methodology: It reports the nurses' experience at delivery care at the obstetrical center. Results: The experience made it possible to give the thematic of early breastfeeding an insight, pointing out this approach at the delivery room. It was shown that this measure is not effectively implemented, being postponed to the post-delivery room. There is prevalence of caesareans in hospitals hindering the promotion of the breastfeeding practice at delivery and birth. Another thing that limits that practice is the assistance attached to the standards, institutional rules, and the culture of biomedical competence. Conclusion: Highlight that hospitals need to develop policies to promote and protect the breastfeeding practice.

Descriptors: Breast feeding; Obstetrical nursing; Humanizing delivery; Women's health.

RESUMEN: Obj etivo: relatar la experiencia de la lactancia materna en la primera hora de vida del recién nacido en un hospital privado de Porto Alegre/RS, abordando sus ventajas y las principales dificultades en la adopción de esta práctica. Metodología: Se trata de un relato de

\footnotetext{
${ }^{1}$ Enfermeira. Especialista em Enfermagem Obstétrica. Enfermeira assistencial do Centro Obstétrico do Hospital Mãe de Deus. Integrante GEMBE/ UFRGS. E-mail: marcirej ane@yahoo.com.br

2 Enfermeira. Especialista em Enfermagem Obstétrica e Enfermagem Neonatal. Enfermeira do Centro Obstétrico do Complexo Hospitalar Santa Casa de Porto Alegre. Integrante GEMBE/UFRGS. E-mail: enffischer@yahoo.com.br

${ }^{3}$ Enfermeira. Doutora em enfermagem. Professora Adjunta do Departamento de Enfermagem Materno Infantil da Universidade Federal do Rio Grande do Sul. Porto Alegre, Rio Grande do Sul, Brasil. E-mail: bonilha.ana@gmail.com
} 


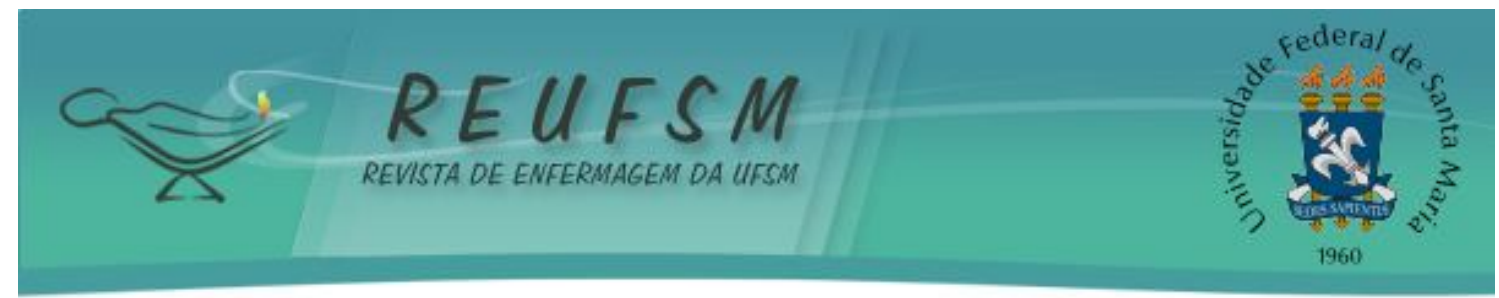

experiencia realizado por las enfermeras durante la atención al parto en el centro obstétrico. Resultados: La experiencia relatada ha permitido visibilizar la temática de la amamentación precoce, señalando la importancia de esta práctica en la sala de parto. Se constató que esta medida no está implementada efectivamente, aplazándose para la sala de recuperación obstétrica. Hay prevalencia de cesáreas en la institución hospitalar, lo que dificulta la promoción de la lactancia materna en el escenario del parto y nacimiento. Otro factor que limita esta práctica es la asistencia vinculada a las normas, a las reglas institucionales y a la cultura de competencia biomédica. Conclusión: Se destaca la necesidad de la institución hospitalar desarrollar políticas de promoción y protección a la lactancia materna.

Descriptores: Lactancia Materna; Enfermería Obstétrica; Parto Humanizado; Salud de la Mujer.

\section{INTRODUÇÃO}

Historicamente, o tema aleitamento materno ressurgiu na década de 70 , com novas discussões, a partir de estudos que mostravam aumento da morbimortalidade infantil, como conseqüência do uso indiscriminado de fórmulas lácteas na alimentação nos primeiros meses de vida. ${ }^{1}$

A partir de 1980 a Organização Mundial da Saúde (OMS) e o Fundo das Nações Unidas para a Infância (UNICEF) envidaram esforços para a instituição de uma política de incentivo à amamentação. Essa política foi implementada pela criação da Iniciativa do Hospital Amigo da Criança (IHAC) por meio dos chamados "dez passos para o sucesso do aleitamento materno. Este evento ocorreu durante um encontro realizado em Florença (Itália), onde se produziu a Declaração de Innocenti, que resgata o direito da mulher de aprender e praticar amamentação com sucesso. ${ }^{2}$

Com destaque ao quarto passo da IHAC, a declaração salienta a necessidade de ajudar as mães a iniciarem a amamentação na primeira meia-hora após o parto, ressaltando que após o nascimento a mãe tem condições de sustentar física e psiquicamente o bebê. Neste momento há favorecimento do vínculo entre a mãe e o bebê. ${ }^{2}$

Em consonância com a temática, pode-se afirmar que o aleitamento materno na primeira hora de vida tem papel fundamental na redução dos índices de morbimortaliade infantil, mais especificamente no componente neonatal, e nas internações hospitalares precoces. ${ }^{3-4}$

o impacto do aleitamento materno na sobrevivência neonatal e infantil foi analisado a partir de dados de mortalidade de 42 países que representam $90 \%$ das mortes do mundo no ano de 2000. A partir do estudo, estimou-se que a amamentação exclusiva durante os seis primeiros meses de vida, seguida do aleitamento continuado dos 06 a 11 meses de idade, era a intervenção mais efetiva para prevenir a mortalidade infantil e que é capaz de prevenir $13 \%$ de todas a mortes em menores de cinco anos. ${ }^{4}$

Para tal, o Ministério da Saúde do Brasil em sua Política Nacional de Promoção, Proteção e Apoio ao Aleitamento Materno busca parcerias com o objetivo de aumentar as taxas de amamentação: Rede Amamenta Brasil, Iniciativa Hospital Amigo da Criança (IHAC), proteção legal ao aleitamento materno, rede brasileira de bancos de leite humano, mobilização social e monitoramento dos indicadores de aleitamento materno no Brasil. ${ }^{5}$

Quanto a prevalência de aleitamento materno nas capitais brasileiras e Distrito Federal, 67,7\% das crianças mamam na primeira hora de vida. ${ }^{6} \mathrm{~J}$ á o percentual de crianças menores de 60 meses, alimentadas com leite materno, conforme Pesquisa Nacional sobre Demografia e Saúde em 2006 (PNDS/2006), é de 43\% ${ }^{6}$

Mesmo com o incentivo à amamentação desenvolvido pelas instituições públicas e privadas no Brasil, esta prática está muito aquém da preconizada pela World Health Organization (WHO), que recomenda o aleitamento exclusivo até os seis primeiros meses, devendo ser prolongado até o segundo ano de vida do lactente. ${ }^{7}$ 


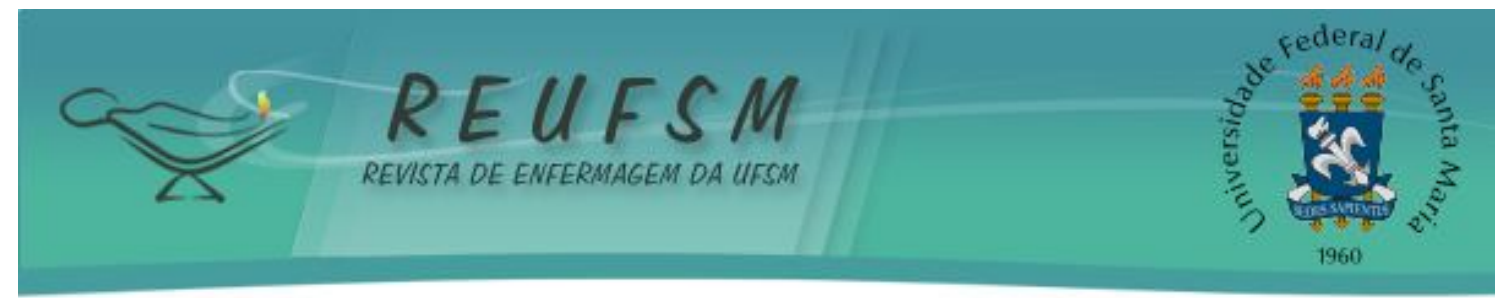

Um estudo realizado na Maternidade do Hospital Israelita Albert Einstein, em 2008, buscou identificar a prevalência e os fatores limitantes da amamentação na primeira hora de vida do recém-nascido. A prevalência de sucesso foi de $74,3 \%$ entre mães e recém-nascidos sadios, e o insucesso, $18,7 \%$ Os principais fatores limitantes identificados foram: alta rotatividade de partos, recusa pela paciente, recusa médica, cansaço por trabalho de parto prolongado, perda de registro de dados. 0 parto operatório e o uso de anestesia não foram fatores impeditivos à amamentação na primeira hora de vida e ao contato pele à pele. ${ }^{8}$

No que tange a importância da amamentação na primeira hora, faz-se necessário que as mães sejam estimuladas pela equipe multidisciplinar à amamentar ainda na sala de parto. Neste cenário, a mulher deve ser respeitada em sua individual idade e especificidade cultural, podendo tornar-se suj eito desta prática. ${ }^{3}$

Cabe aos profissionais de enfermagem a função de facilitadores, no que tange a prática da amamentação precoce, especialmente na orientação e auxílio durante as práticas institucionalizadas na sala de parto.

0 enfermeiro pode atuar junto aos demais profissionais da enfermagem e aos demais profissionais da saúde, como por exemplo, os médicos, buscando informá-los, sensibilizá-los e integrá-los ao programa de incentivo, promoção, e apoio à amamentação na primeira hora de vida. Para isso deve-se buscar o conhecimento científico, desenvolver a competência técnica e de comunicação. ${ }^{8}$

Este estudo tem como objetivo, relatar a experiência da amamentação na primeira hora de vida do recém-nascido, abordando suas vantagens e as principais dificuldades para a adoção desta prática.

\section{MÉTODO}

Trata-se de um relato de experiência acerca da amamentação na primeira hora do nascimento em um hospital privado de Porto Alegre/ RS, numa abordagem de humanização no centro obstétrico, realizada pelas enfermeiras em meio às atividades assistenciais e gerenciais de enfermagem.

A partir do relato da experiência e da discussão são apresentadas contribuições para a prática assistencial mediante suporte científico em literatura pertinente.

\section{CARACTERIZAÇÃO DO CENTRO OBSTÉTRICO}

A maternidade, foco do presente relato, presta atendimento há mais de duas décadas junto a mulheres usuárias de planos de saúde ou particulares, com equipes médicas, de sua livre escolha, desde que cadastradas no Hospital. A área materno infantil corresponde à maternidade, centro obstétrico, intensivismo, lactário e sala de recuperação pós parto.

A estrutura do centro obstétrico compreende quatro salas de preparo, duas salas de parto pré-parto, parto e puerpério imediato (PPP), duas salas de cesariana e sala de recuperação obstétrica composta por quatro leitos. Além disso, segue protocolos clínicos internacionais, buscando assegurar padrão de qualidade médico-assistencial e a segurança da paciente e do recém-nascido.

Conta com uma equipe de profissionais especialistas: enfermeiras obstétricas, plantão médico obstétrico e neonatologistas.

As salas de parto são do tipo PPP, ou seja, pré-parto, parto e puerpério. As gestantes que internam em trabalho de parto são encaminhadas para este ambiente, no qual permanecem até 0 nascimento de seu bebê. A cama de parto é eletrônica e se adapta às diferentes posições, conforme o plano de parto da paciente seja ele posição litotomia, cócoras, recostada entre outras. 


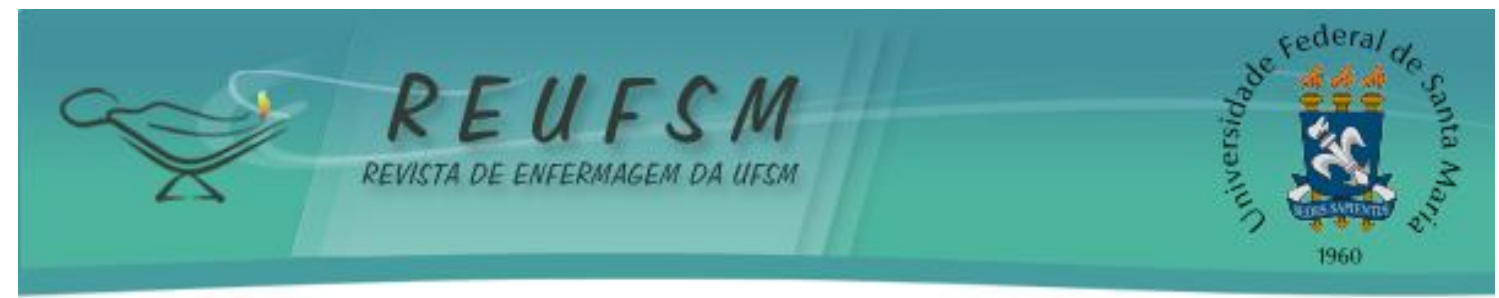

Ao chegar ao Centro Obstétrico, a gestante é avaliada pelo profissional médico que está de plantão, que após faz contato com o médico assistente. A enfermeira juntamente com sua equipe faz a admissão da paciente, monitorização com cardiotocofetal e atendimento da prescrição médica.

Em anexo a sala de parto, localiza-se a sala de admissão do recém-nascido, com berços aquecidos, parede de gases, carro de reanimação, bancada para banho, balança para peso, entre outros. Igualmente ao lado da sala de cesárea, localiza-se outra de admissão de recém-nascido, disposta com os mesmos equipamentos.

Cabe salientar que quando a cesárea é eletiva, a paciente chega por volta de uma hora antes do horário do procedimento para o preparo pré-cirúrgico.

A equipe de profissionais envolvida no evento do nascimento é composta pelo obstetra, pediatra, enfermeira e técnicos de enfermagem.

\section{RELATO DE EXPERIÊNCIA}

Durante todo o ciclo-gravídico-puerperal, a mulher precisa ser atendida em sua integralidade, por uma equipe engajada e humanizada, já que está repleta de dúvidas, ansiedades, frustrações e medos. ${ }^{9}$

Os profissionais de enfermagem por sua vez precisam estar qualificados para prestar auxílio neste período com grandes transformações, assim a atenção obstétrica e neonatal, prestada pelos serviços de saúde, deve ter como características essenciais a qualidade e a humanização.

No cenário do parto e nascimento, a mulher é atendida com garantia do seu bem estar e do seu neonato. São incorporadas condutas acolhedoras, sem intervenções técnicas desnecessárias, com ações de cuidado que integram promoção, proteção e atenção à saúde da gestante e do recém-nascido. A gestante é acolhida e compreendida frente seus significados de gestação e o pai estimulado a participar do evento do nascimento e dos cuidados com o recém-nascido. ${ }^{10}$

Mesmo no espaço privado, a atenção humanizada pode ser priorizada. Neste sentido a humanização do parto e nascimento atualmente preconizados pode ser implementada, com vistas à amamentação na primeira hora de vida.

Contudo, percebe-se na prática profissional das autoras que embora a humanização do parto e nascimento seja uma temática discutida com freqüência nos eventos e fóruns de amamentação, é pouco presente nas ações de cuidados dos profissionais de saúde.

Ao nascer, o bebê é recebido pelo pediatra e envolto em um campo seco e aquecido, apresentado a mãe e transferido para a sala de admissão do recém-nascido, onde são realizados os primeiros cuidados como: secar e estimular, troca do campo úmido por outro seco e aquecido; colocar cord-clamp; identificar o RN; realizar conferência do nome correto da mãe e mostrar ao acompanhante (colocar pulseira com o nome da mãe) em membro superior; pesar o RN; realizar medidas antropométricas. 0 médico avalia 0 índice de Apgar.

Após ter recebido os cuidados descritos e ter sido avaliado pelo pediatra, o bebê retorna à mãe onde permanecerá por alguns minutos e depois de socializado junto à família que normalmente aguarda ansiosa por notícias e pela chegado do bebê. Em seguida retorna a sala de admissão onde é colocado no berço aquecido.

É imprescindível que ocorra o encorajamento da amamentação imediatamente após o nascimento a fim de manter e ocasionar a liberação de ocitocina, promover a involução uterina de modo mais rápido, incentivar a amamentação, realizar a avaliação das mamas e orientar quanto ao processo de amamentação. ${ }^{11}$

O período imediato após o parto constitui o momento de maior sensibilidade da puérpera, sendo assim, o incentivo a amamentação na primeira hora de vida do recém- 


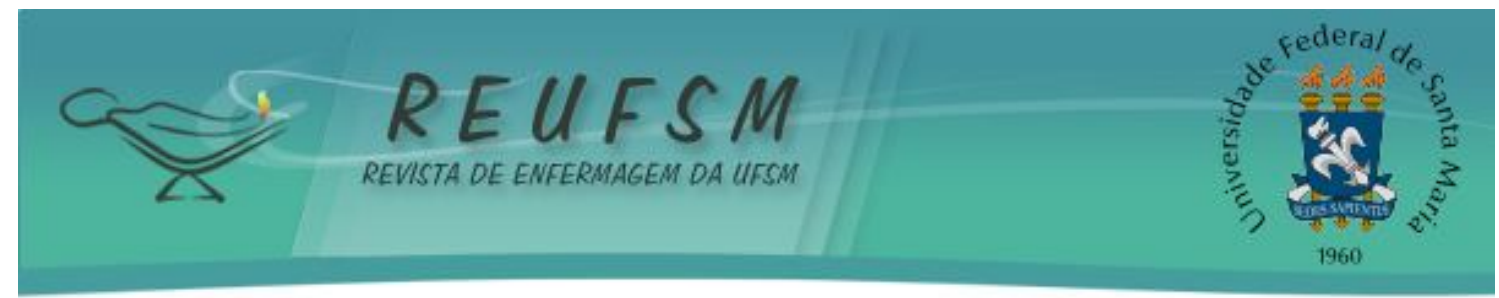

nascido, constitui uma medida que deve ser estimulada pelos profissionais para proporcionar a experiência de amamentação à mulher e promover 0 vínculo afetivo. ${ }^{12}$

0 contato com a pele da mãe durante a amamentação, imediatamente após 0 nascimento, promove além do aquecimento e do conforto, um ambiente ideal para a adaptação do recém-nascido à vida extra-uterina, promovendo uma precoce interação mãe-bebê. ${ }^{13}$

Cabe a equipe de saúde oferecer apoio qualificado as mães durante a primeira mamada, e quando necessário também nas mamadas seguintes, para assegurar que 0 recém-nascido tenha uma sucção ef etiva. ${ }^{4}$

São inúmeros os benefícios imediatos do aleitamento materno exclusivo logo após o parto. Para o recém- nascido, o colostro conhecido como a "primeira vacina" garante a capacidade contra infecções, como por exemplo, para enterocolite necrosante, infecções do trato gastrointestinal e respiratório, alergias, septicemia e meningites, além de estar sempre pronto na temperatura ideal para a criança. ${ }^{4}$

Igualmente, o aleitamento materno imediato após o parto é benéfico para a mãe, já que a sucção estimula a liberação de ocitocina endógena, que induz a contração uterina, prevenindo hemorragias puerperais, principal causa de mortalidade materna no mundo. Além disto, o ato de amamentar age como protetor nos transtornos de estado de animo materno e favorece a formação do vínculo afetivo entre a mãe e o bebê. ${ }^{4}$

Observa-se que nem sempre os sentimentos e os desejos das mulheres são respeitados na sala de parto. Nesse momento os profissionais podem ser determinantes na amamentação, permitindo ou não que esta prática seja implementada.

0 enfermeiro que atua no Centro Obstétrico deve se posicionar frente a esta temática trabalhando com a sua equipe, pois os profissionais tem grande potencial para realizar mudanças e implementar novas práticas que são essenciais para a mãe e o bebê.

O contato pele-a-pele e a amamentação na primeira hora de vida são incentivados e garantidos à mãe e ao bebê na sala de parto. Para tal, a equipe de enfermagem deve desenvolver competências e habilidades em aleitamento materno para realizar intervenções adequadas e superar as possíveis barreiras à amamentação.

Acredita-se que o enfermeiro é capaz de modificar práticas habituais na sala de parto, iniciando por sua presença e postura no nascimento. A educação continuada junto à equipe de enfermagem é indispensável, contribuindo para a responsabilidade e o compromisso com o cuidado humanizado.

Autores $^{3}$, afirmaram que o principal fator que contribui para a não implementação da amamentação na primeira hora pode ser o indicativo de que as mães têm pouco ou nenhum poder de decisão para amamentar seus filhos na primeira hora de vida, ficando reféns das práticas institucionais vigentes nas maternidades e dos profissionais envolvidos no parto. ${ }^{3}$

Vale salientar que as cesáreas atingem cerca de $90 \%$ dos partos realizados nesta instituição hospitalar.

No Brasil, as taxas de cesariana são mais altas entre as mulheres com melhores condições socioeconômicas, as de etnia branca e aquelas tendo parto em hospitais privados, ou seja, entre as mulheres potencialmente de menor risco obstétrico. Esta incidência está relacionada também à existência de uma prática obstétrica e da atenção ao parto na qual a cesárea prévia é a determinante na escolha do tipo de parto. ${ }^{15}$

Frente a esta situação, percebe-se que a equipe de enfermagem atém-se as normas e rotinas, com a preocupação em atender a prescrição médica e os protocolos institucionais.

0 ambiente hospitalar tem sido considerado bastante restrito, em termos de práticas educativas humanizadas, atendo-se à burocracia do cuidado, centrado nas normas, às regras institucionais e ao modelo biomédico. Esta característica compromete o cuidado, limitando a equipe de enfermagem na implementação de práticas efetivas de cuidado, de troca de saberes e de educação em saúde no evento do parto e nascimento. 


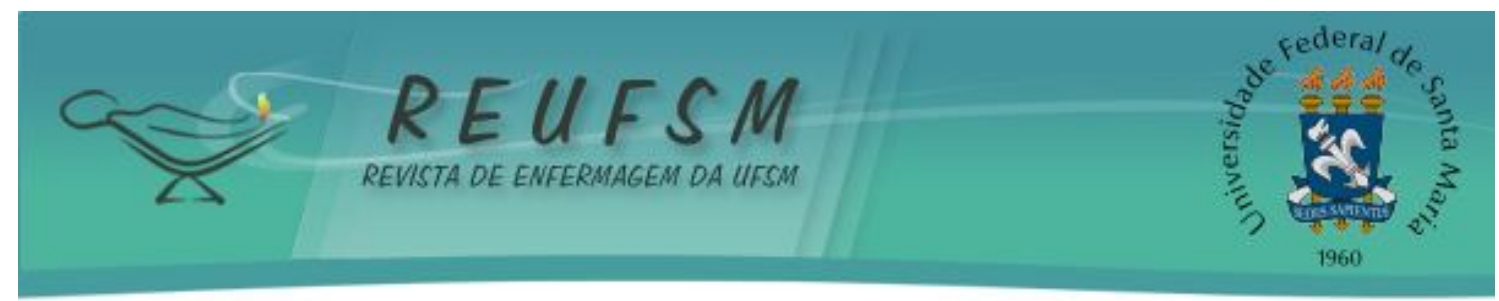

Contudo, a OMS preconiza adiar, pelo menos durante a primeira hora de vida, qualquer procedimento rotineiro de atenção ao recém-nascido que separe a mãe de seu bebê, com o objetivo de permitir o contato pele-a-pele ininterrupto entre a mãe e o bebê. Como exemplo, tem-se a credeização ou prevenção da oftalmia neonatal, que pode se postergada para após os primeiros sessenta minutos de vida da criança, a adoção desta prática incentiva e promove o início da amamentação na primeira hora de vida.

Destaca-se que é importante oferecer apoio qualificado às mães durante a primeira mamada e, quando necessário, também nas mamadas seguintes, para assegurar que o recém-nascido tenha uma boa sucção e mame efetivamente.

Quanto ao cuidado com o neonato, busca-se, na primeira hora manter a atividade respiratória do recém-nascido ( 35 a $60 \mathrm{mrpm}$ ), realizando desobstrução das vias aéreas superiores se necessário; manter atividade circulatória (120 a 160 bpm), com aquecimento adequado; manter 0 ambiente térmico do RN temperatura $(36,8$ a $37,5 \circ C$. $)$. Após são realizados os demais procedimentos como: administração de vitamina K, vacina para hepatite B entre outros.

0 recém-nascido permanece acompanhado pelo pai neste período enquanto 0 obstetra finaliza o atendimento à mulher. Após, a mãe é transferida para a sala de recuperação obstétrica onde é monitorizada.

Assim que o bebê tiver recebido os cuidados de rotina, inclusive o banho, retorna para a mãe que 0 aguarda na sala de recuperação.

Neste momento é novamente estimulada a amamentação e a formação de vínculo através do contato com a pele da mãe. O pai é orientado a permanecer o tempo todo com a mãe e o bebê.

Entre outros profissionais de saúde que podem realizar essa aproximação da mãebebê e família, destaca-se o enfermeiro, que tem várias oportunidades de contato com gestantes, mães e seus recém-nascidos, nos diferentes níveis de atenção à saúde. ${ }^{2}$

0 enfermeiro é um profissional que se faz presente durante todo o ciclo gravídicopuerperal. È habilitado por legislação a conduzir pré-natal de baixo risco, solicitar exames de rotina e complementares, executar consulta de enfermagem transmitindo neste momento apoio e confiança para que a gestante possa se fortalecer e conduzir com mais autonomia a gestação e o parto, e as decisões em relação ao cuidado com o seu filho, incluindo-se entre estas a amamentação.

Durante o período do puerpério o enfermeiro aplica seus conhecimentos para a prática dos cuidados com a mãe e neonato. Dispõe de orientações para o auto-cuidado, cuidado com o recem nascido e planejamento do atendimento de enfermagem.

Assim, entende-se que a enfermagem pode ser um agente de mudanças, respeitando a cultura, esclarecendo dúvidas relacionadas a aspectos que podem ser prejudiciais a mulher e provocar medo e ansiedade, por meio de promoção, de orientação no pré-natal, puerpério e puericultura com vistas aos cuidados da mulher, criança e família. ${ }^{14}$

\section{CONSIDERAÇÕES FINAIS}

Este relato de experiência permitiu refletir sobre a amamentação na primeira hora de vida e perceber que esta prática pode ser realizada de modo mais efetivo pela equipe multiprofissional.

Acredita-se que por a instituição privada com alta ocorrência de cesarianas pode dificultar esta prática, uma vez que o cenário se torna mais complexo.

Neste contexto, 0 atendimento de enfermagem segue o modelo biomédico tornando visível a preocupação da equipe em implementar as rotinas do cuidado do recémnascido e em atender a prescrição médica, como a aplicação da vitamina $k$, hepatite $b, 0$ banho, entre outros. 


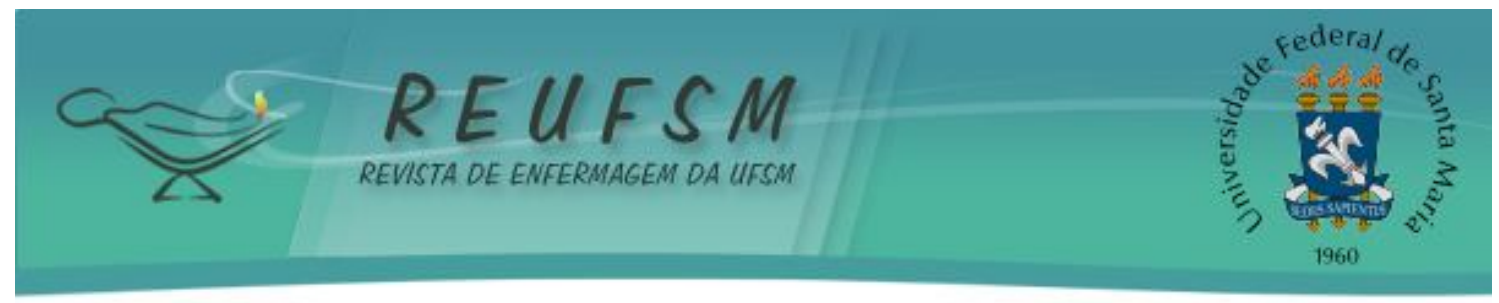

A mudança de atitude do profissional de saúde, com a integração e valorização da mãe, do seu filho e do seu acompanhante, pode facilitar a operacionalização desta prática, de modo que esta seja pautada pelos princípios da humanização, com respeito e acolhimento daqueles que são cuidados.

A responsabilidade dos profissionais de enfermagem é determinante para garantir à mãe e ao recém-nascido o direito à amamentação na primeira hora de vida. Contudo, torna-se indispensável sensibilizar os gestores e a administração hospitalar para a promoção desta prática.

Sugere-se que a instituição de saúde invista em políticas que promovam a amamentação na primeira hora de vida do recém-nascido como a Iniciativa Hospital Amigo da Criança (IHAC) e que sua prática possa ser estimulada e iniciada na sala de parto.

\section{REFERENCIAS}

1. Melo AMCA, Cabral PC, Albino E, Moura LMD, Menezes AEB, Wanderley LG. Conhecimentos e atitudes sobre al eitamento materno em primíparas da cidade do Recife, Pernambuco. Rev Bras Saúde Mater Infant. 2002; 2(2): 137-42.

2. Barbosa V, Orlandi FS, Dupas G, Beretta MIR, Fabbro MRC. Aleitamento materno na sala de parto. Ciênc Cuid Saúde. 2010; 9(2): 366-73.

3. Boccolini CS, Carvalho ML, Oliveira MIC, Vasconcellos AGG. Fatores associados à amamentação na primeira hora de vida. Rev saúde pública. 2011; 45(1): 69-78.

4. Organização Panamerica de Saúde. Além da sobrevivência: práticas integradas de atenção ao parto, benéficas para nutrição e a saúde de mães e crianças. Washington, D.C.: OPAS; 2007.

5. Ministério da Saúde [homepage na internet]. Brasília: Promoção, proteção e apoio ao aleitamento materno [Acesso em 23 de dezembro de 2010 dez]. Disponível em: http:// portal. saude.gov. br/ portal/ saude/ area. cfm?id_area $=1460$.

6. Ministério da Saúde (BR), Secretaria de Atenção a Saúde, Departamento de Ações Programáticas e Estratégicas. II pesquisa de prevalência de aleitamento materno nas capitais brasileiras e Distrito Federal. Brasília (DF): Ministério da Saúde; 2009.

7. World Health Organization, Collaborative Study Team on the Role of Breastfeeding on the Prevention of Infant Mortality. Effect of breastfeeding on infant and child mortality due to infections diseases in less developed countries: a pooled analysis. Lancet. 2000;355(9202):451-5.

8. Pillegi MC, Policastro A, Abramovici S, Cordioli E, Deutsch AD. A amamentação na primeira hora de vida e a tecnologia moderna: prevalência e fatores limitantes. Einstein. 2008; 6(4): 467-72.

9. Meneghin AC, Tapia CEV. Ciclo gravídico puerperal e a enfermagem: proposta de protocolo. Rev Enferm UFPE On Line [periódico na Internet]. 2008 [citado 2010 dez 23]; 2(4): 445-53. Disponível em: http:/ / www. ufpe. br/ revistaenfermagem/ index. php/ revista/ article/ view/332/ pdf_407. doi: 10.5205/ reuol.332-11493-1-LE. 0204200816.

10. Brasil. Ministério da Saúde. Pré-natal e puerpério: atenção qualificada e humanizada: manual técnico. Brasília: Ministério da saúde; 2005.158p. 


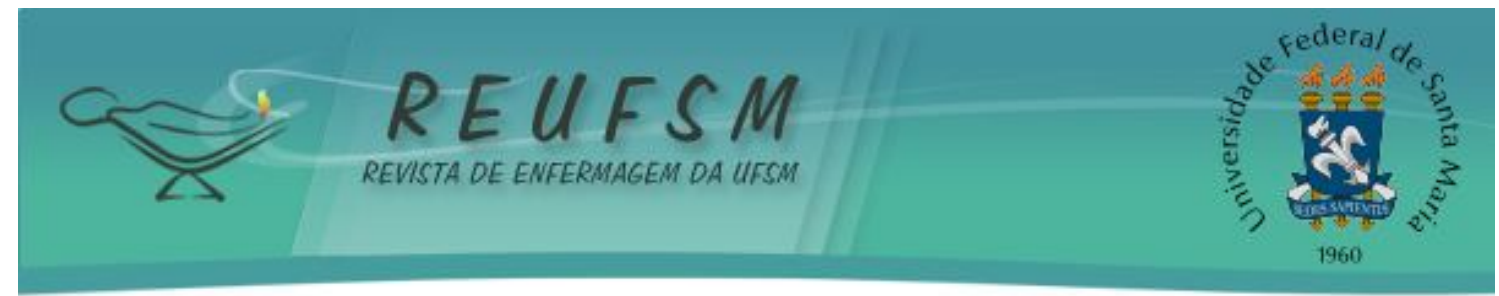

11. Stetson B. Mudanças fisiológicas no puerpério. In: Lowdermilk DL, Perry SE, Bobak IM. 0 cuidado em enfermagem materna. 5a ed. Porto Alegre: Artmed; 2002. p. 415-423.

12 Ballone, G]. Depressão pós-parto [homepage na Internet]. São Paulo: Sociedade Paulista de Psiquiatria Clínica; 2002 [citado em 24 de dez 2010]. Disponível em: বttp:// wwwpsiqweb. med.br/ sexo/ posparto.html>.

13. Mikiel-Kostyra K., Mazur J., Boltruszko I. Effect of early skin-to-skin contact after delivery on duration of breastfeeding: a prospective cohort study. Acta paediatr. 2002; 89(12): 1301-6.

14. Marques DM, Pereira AL. Amamentar: sempre benefícios, nem sempre prazer. Ciênc Cuid Saúde. 2010; 9(2): 214-19.

15. Freitas PF, Drachler ML, Leite JCC, Grassi PR. Desigualdade social nas taxas de cesariana em primárias no Rio Grande do Sul. Rev saúde pública. 2005; 39(5): 761-7.

Data de recebimento: 13/04/2011

Data de aceite: $21 / 07 / 2011$

Contato com autora principal: Márcia Rejane Strapasson

Endereço postal: Rua Santo Antônio, 306. Bairro Floresta, Porto Alegre, RS.

CEP: $90220-010$

E-mail: marcirejane@yahoo.com.br 